\title{
Politikkoordination im deutschen Bundesstaat: Wandel in den Arbeitsstrukturen?*
}

\author{
Christina Zimmer
}

Der deutsche Bundesstaat zeichnet sich durch eine funktionale Aufgabenverteilung aus, bei der die Gesetzgebung weitgehend beim Bund liegt, der Vollzug dagegen bei den Ländern. Die Länder wiederum haben über den Bundesrat - auch nach der Föderalismusreform von 2006 - weitreichende Mitspracherechte an der Bundesgesetzgebung. Ein solcher „intrastaatlicher Föderalismus " ${ }^{1}$ erfordert ein erhebliches Maß an Koordination nicht nur zwischen den staatlichen Ebenen, sondern auch der Länder untereinander. Während bei intergouvernementalen Zwangsverhandlungen (Zustimmungsgesetzgebung, Gemeinschaftsaufgaben) Kooperation unabdingbar ist, da nur ein gemeinsames Ergebnis möglich und dann auch für alle Beteiligten bindend ist, koordinieren die Länder, meist unter Beteiligung des Bundes, wichtige Bereiche ihrer Politik freiwillig. Dabei ist die Kooperation nicht verfassungsrechtlich normiert, und letztlich steht jedes Land selbst in der Verantwortung. Dennoch streben die Länder auch hier regelmäßig gemeinsame Lösungen an, was mit der in Deutschland schon seit dem Wilhelminischen Reich dominierenden unitarischen Kultur $^{2}$ erklärbar ist, in jüngerer Zeit ergänzt von Anforderungen der EU in Bezug auf einheitliche Berichtsstandards und bei der Umsetzung von Richtlinien.

Bund und Länder haben entsprechend die horizontale und vertikale Koordinierung auch institutionell ausgebaut: Konferenzen der Ministerpräsidenten beraten vierteljährlich politische Leitlinien und Grundsatzfragen der Bundes- und Landespolitik; zweimal im Jahr finden im Anschluss Gespräche mit der Bundeskanzlerin statt. Die ressortweise Abstimmung der politischen Akteure wird in den Fachministerkonferenzen gebündelt. ${ }^{3}$ Darüber hinaus haben diese auf der Ebene der nachgeordneten Ministerial- und Fachverwaltungen eine große Zahl von Arbeitsgremien ${ }^{4}$ eingerichtet. Die „Fülle kooperativer Erscheinungen “5 macht eine Übersicht und Kategorisierung zum Teil äußerst schwierig. Hauptaufgaben der Gremien sind die Sicherstellung eines einheitlichen Vollzugs, Abstimmungen in Gesetzge-

* Dieser Beitrag basiert auf einem Vortrag im Rahmen des 24. Wissenschaftlichen Kongresses der DVPW im Forum für Junge Staats- und Verwaltungswissenschaft (FoJuS) im September 2009 an der Christian-Albrechts-Universität zu Kiel. Dem dortigen Fachpublikum danke ich ebenso wie Arthur Benz, dem Betreuer meiner Dissertation, und den Kollegen an der FernUniversität in Hagen für wertvolle Anregungen und Kommentare.

1 Vgl. Jörg Broschek, Der kanadische Föderalismus: Eine historisch-institutionalistische Analyse, Wiesbaden 2009.

2 Vgl. Konrad Hesse, Der unitarische Bundesstaat, Karlsruhe 1962; Gerhard Lehmbruch, Der unitarische Bundesstaat in Deutschland. Pfadabhängigkeit und Wandel, in: Arthur Benz / ders. (Hrsg.), Föderalismus. Analysen in entwicklungsgeschichtlicher und vergleichender Perspektive, PVSSonderheft 32, Wiesbaden 2002, S. $53-110$.

3 Als vorerst letzte der derzeit 18 Fachministerkonferenzen wurde 2008 die Integrationsministerkonferenz eingerichtet.

4 Der Begriff „Gremien“ wird im Folgenden als übergeordnete Bezeichnung für Ausschüsse, Kommissionen, Arbeitsgemeinschaften, Arbeitskreise etc. verwendet.

5 Michael Bothe, Die Kompetenzstruktur des modernen Bundesstaates in rechtsvergleichender Sicht, Berlin / New York / Wien 1977, S. 278. 
bungsverfahren sowie die Vorbereitung von Sitzungen der Ministerkonferenzen. Es stehen aber auch strategische Vorüberlegungen und politische Grundsatzfragen auf der Tagesordnung. Das ungefähre Ausmaß der Gremienlandschaft deutet sich beim Versuch einer Quantifizierung an: So ermittelte beispielsweise im Jahr 1973 die Stadt Hamburg 1.074 Gremien der „Zwischenländerkooperation“6; eine Erhebung des Nordrhein-Westfälischen Landtags wies 1989 lediglich circa 450 bis 470 Arbeitsgremien aus ${ }^{7}$, während Baden-Württemberg Ende der 1990er Jahre wieder 928 länderübergreifende Gremien mit eigener Beteiligung zählte. ${ }^{8}$

Die Arbeitsstrukturen der horizontalen und vertikalen Koordinierung werden in Literatur und Praxis durchaus kritisch betrachtet. Die Argumente reichen von einer allgemeinen Kritik an der „Kleinstaaterei“ im Föderalismus und dem damit verbundenen Koordinationsaufwand einerseits beziehungsweise der Sorge um die Aufgabe föderaler Eigenständigkeit andererseits 9 , über die Problematik der demokratischen Legitimation von Politik im Exekutivföderalismus ${ }^{10}$ bis hin zur Diskussion um Effizienzsteigerung, Entbürokratisierung und Verwaltungsmodernisierung, die in Deutschland bereits Mitte der 1970er Jahre ihren Anfang nahm. ${ }^{11}$ Mit der zunehmenden Verschuldung der öffentlichen Haushalte wurden Leitideen wie Bürokratieabbau und „schlanker Staat" um das Ziel der Haushaltskonsolidierung ergänzt, um über Einsparungen beim Personal die fiskalischen Probleme abzumildern. Nicht zuletzt stand dann bei den Verhandlungen zur Föderalismusreform I im Jahr 2004 das Ziel der Entflechtung des Föderalismus an erster Stelle. ${ }^{12}$

Damit ist in groben Zügen der Kontext des Reformdiskurses beschrieben, in dem die Ministerpräsidenten zum wiederholten Mal angestrebt haben, die Arbeitsgremien der Fachministerkonferenzen zu reduzieren. Mit formalen Beschlüssen forderte die Ministerpräsidentenkonferenz (MPK) die Fachminister in den Jahren 1998 und noch einmal 2004 auf, eine deutliche Verringerung der Gremienzahl zu erreichen und die bundesstaatliche Koordination stärker projektbezogen und in zeitlich befristeten Gremien durchzuführen. ${ }^{13}$ Hier

6 Jost Pietzcker, Landesbericht Bundesrepublik Deutschland, in: Christian Starck (Hrsg.), Zusammenarbeit der Gliedstaaten im Bundesstaat. Landesberichte und Generalbericht der Tagung für Rechtsvergleichung 1987 in Innsbruck, Baden-Baden 1988, S. 17 - 76, S. 25.

7 Vgl. Heiderose Kilper / Roland Lhotta, Föderalismus in der Bundesrepublik Deutschland, Opladen 1996, S. 132.

8 Vgl. Gesamtübersicht der Vertretung der Ministerien des Landes Baden-Württemberg in länderübergreifenden Gremien und Arbeitsgruppen vom 2. Juni 1998.

9 Vgl. Hartmut Klatt, Interföderale Beziehungen im kooperativen Bundesstaat - Kooperation und Koordination auf der politischen Leitungsebene, in: Verwaltungsarchiv, 78. Jg. (1987), H. 2, S. $186-206$.

10 So zum Beispiel Wolfgang Zeh, Bund-Länder-Kooperation und die Rolle des Landesparlaments, in: Hartmut Klatt (Hrsg.), Baden-Württemberg und der Bund, Stuttgart 1989, S. 119 - 146.

11 Vgl. Jörg Bogumil / Werner Jann, Verwaltung und Verwaltungswissenschaft in Deutschland, Wiesbaden 2009.

12 Vgl. Arthur Benz, Föderalismusreform in der „Entflechtungsfalle“, in: Europäisches Zentrum für Föderalismus-Forschung (Hrsg.), Jahrbuch des Föderalismus, Bd. 8, Baden-Baden 2008, S. 180 -190 .

13 Der Beschluss der MPK vom 17. Juni 2004 (TOP 7) lautete: „1. Die Regierungschefs der Länder halten zur Entbürokratisierung den weiteren Abbau länderübergreifender Gremien und Arbeitsgruppen für notwendig. Sie versprechen sich vom weiteren Abbau überregionaler Gremien eine effektivere Nutzung verfassungsrechtlicher und einfachgesetzlicher Gestaltungsspielräume. 2. Die Regierungschefs der Länder sprechen sich für die Auflösung der länderübergreifenden Gremien 
knüpft dieser Beitrag an, da die von der MPK in diesem Zusammenhang veröffentlichten Zahlen in der Tat auf eine starke Dynamik hinweisen. ${ }^{14}$ Im Folgenden wird zunächst dargestellt, in welcher Form beziehungsweise in welchem Umfang die Vorgaben der MPK von den jeweiligen Fachministerkonferenzen umgesetzt worden sind und welche Unterschiede dabei insbesondere zwischen Politikfeldern bestehen. Für solche Bereiche, die eine deutliche Gremienreduktion erfahren haben, ist daran anschließend zu überlegen, welche Auswirkungen auf die Koordination im Bundesstaat zu erwarten sind. Da als Folge von Gremienreduktion einerseits von einer Stärkung politisierter Akteure an der Schnittstelle von Politik und Verwaltung - und damit von einer Politisierung der Koordinationsprozesse auszugehen ist, kommen theoretische Überlegungen bezüglich der Spannungen zwischen Parteienwettbewerb und Bundesstaatlichkeit zum Tragen. Eine verstärkte Interessenorientierung auf Kosten von Problemlösungsorientierung könnte die bundesstaatliche Koordination verändern und die fachlichen Abstimmungsprozesse schwächen. Andererseits basiert die bundesstaatliche Zusammenarbeit seit jeher auf starken sektoralen Verwaltungsnetzwerken, die nur schwer zu überwinden sein dürften. Dort könnte es den beteiligten Akteuren gelingen, formale Veränderungen zu kompensieren oder zu umgehen, so dass sich Koordinationsprozesse im Ergebnis kaum verändern würden.

\section{Theoretische Überlegungen zum Gremienabbau}

\subsection{Politisierung}

Aus den Ergebnissen der Elitenforschung in der Verwaltungswissenschaft lässt sich die grundlegende Annahme ableiten, dass Gremienreduktion - zumindest in einem signifikanten Ausmaß - zur Politisierung von Koordination führt beziehungsweise diese verstärkt. Danach wird Koordination vermehrt von der Arbeitsebene auf politische Akteure im engeren Sinne (Staatssekretäre, Minister) verlagert oder konzentriert sich bei den administrativen Eliten innerhalb der Verwaltung. In der Regel gelten die beamteten Staatssekretäre und die Abteilungsleiter als politisierte Verwaltungsakteure; häufig werden auch die Unterabteilungsleiter einbezogen. ${ }^{15}$ Die erste Hypothese lautet also: Gremienreduktion verstärkt die Politisierung von Koordination im deutschen Bundesstaat (Politisierungsthese).

und Arbeitsgruppen bis zum 30. April 2005 aus, vorbehaltlich solcher Gremien, deren unabweisbare Notwendigkeit von den Fachministerkonferenzen rechtzeitig bis zur MPK im April 2005 erläutert wird. Die Regierungschefs der Länder sprechen sich dafür aus, notwendige Abstimmungen nach Möglichkeit künftig nicht in institutionalisierten Gremien auf Dauer, sondern projektund anlassbezogen zeitlich befristet durchzuführen." Senatskanzlei Berlin, http://www.berlin.de/ rbmskzl/dokumentation/mpk/ergebnisse140405.html (Abruf am 14. Juli 2010).

14 Nach Angaben der MPK wurde die Zahl länderübergreifender Gremien und Arbeitsgruppen von über $900 \mathrm{im} \mathrm{Jahr} 1998$ auf $210 \mathrm{im}$ Jahr 2005 reduziert (MPK am 14. April 2005, TOP 3.1).

15 Vgl. Hans-Ulrich Derlien, Verwaltung zwischen Berufsbeamtentum und Parteipolitik. Personalrekrutierung und Personalpatronage im öffentlichen Dienst, in: Politische Bildung, 21. Jg. (1988), H. 2, S. 57 - 72; Renate Mayntz / Hans-Ulrich Derlien, Party Patronage and Politicization of the West German Administrative Elite 1970-1987 - Toward Hybridization?, in: Governance: An International Journal of Policy and Administration, 2. Jg. (1989), H. 4, S. 384 - 404; Katja Schwanke / Falk Ebinger, Politisierung und Rollenverständnis der deutschen Administrativen. Elite 1970 bis 2005 - Wandel trotz Kontinuität, in: Jörg Bogumil / Werner Jann / Frank Nullmeier (Hrsg.), Politik und Verwaltung, PVS-Sonderheft 37, Wiesbaden 2006, S. $228-249$. 
Im Zusammenhang mit der Gremienreduktion sind insbesondere die formale und die funktionale Politisierung von Bürokraten relevant. ${ }^{16}$ Unter formaler Politisierung wird die (Um-)Besetzung von Ämtern der Führungsebene der Ministerialverwaltungen aufgrund politischer Kriterien verstanden, wobei dies rechtlich nur für die Politischen Beamten zulässig ist. Damit ist sie in den Landesverwaltungen für die Ebene der Abteilungsleiter formal ausgeschlossen, da diese dort in der Regel nicht den Status des Politischen Beamten besitzen. Dennoch haben Studien zur parteipolitischen Bindung der administrativen Eliten auch für die Landesverwaltungen einen hohen Anteil parteipolitischer Bindung festgestellt. ${ }^{17}$ Diese begünstigt auch - ist aber keine Voraussetzung für - die funktionale Politisierung von Verwaltungseliten, das heißt ihre Sensibilität und Responsivität bezüglich des politischen Willens der Regierung beziehungsweise der jeweiligen Hausspitze, auch wenn dieser Wille nicht explizit formuliert wird. Die Dimension der funktionalen Politisierung wird seit den 1970er Jahren in empirischen Studien beschrieben. ${ }^{18}$ Dagegen ist zwar das Rollenverständnis von Beamten unterhalb der Leitungsebene ebenfalls als politisch sensibel einzustufen, sie verstehen sich jedoch ausdrücklich als unparteiische Experten. ${ }^{19}$

\subsection{Schwächung von Koordination}

Mit der Politisierungsthese ist bisher nur ein Mechanismus ${ }^{20}$ umschrieben, über den Gremienreduktion zu Veränderungen in der bundesstaatlichen Koordinierung führt. Zu den möglichen Auswirkungen einer Politisierung hat Gerhard Lehmbruch ${ }^{21}$ in seiner Analyse des Parteienwettbewerbs im Bundesstaat das Grundmuster der Argumentation vorgezeichnet. Dem deutschen Regierungssystem ist demnach eine Blockadegefahr immanent, die auf den je unterschiedlichen Handlungslogiken und institutionellen Anforderungen der parlamentarischen und bundesstaatlichen Arenen beruht. In Letzterer gilt die Logik intergouvernementaler Verhandlungen und Kooperation; die parlamentarische Arena wird dagegen von dualistischer Konkurrenz dominiert. Im polarisierten Parteienwettbewerb müssen die Akteure eine Konfliktstrategie fahren, um sich gegenüber dem Wähler profilieren zu können. In der bundesstaatlichen Arena bleiben sie jedoch an die Spielregeln des Aushandelns

16 Vgl. Katja Schwanke / Falk Ebinger, a.a.O. (Fn. 15).

17 Vgl. Hans-Ulrich Derlien, Öffentlicher Dienst im Wandel, in: Klaus König (Hrsg.), Deutsche Verwaltung an der Wende zum 21. Jahrhundert, Baden-Baden 2002, S. 229 - 253.

$18 \mathrm{Vgl}$. Bärbel Steinkemper, Klassische und politische Bürokraten in der Ministerialverwaltung der Bundesrepublik Deutschland. Eine Darstellung sozialstruktureller Merkmale unter dem Aspekt politischer Funktionen der Verwaltung, Bonn 1974; Renate Mayntz / Fritz W. Scharpf, PolicyMaking in the German Federal Bureaucracy, Amsterdam 1975; Robert D. Putnam, The Comparative Study of Political Elites, Englewood Cliffs 1976.

19 Diese Differenzierung haben Renate Mayntz und Fritz Scharpffür die Bundesverwaltung herausgearbeitet. Vgl. dies., a.a.O. (Fn. 18), S. $95-97$.

20 Vgl. zum Begriff des Mechanismus die Übersicht bei John Gerring, Review Article: The Mechanismic Worldview. Thinking Inside the Box, in: British Journal of Political Science, 38. Jg. (2008), H. 1, S. $161-179$.

21 Vgl. Gerhard Lehmbruch, Parteienwettbewerb im Bundesstaat, Stuttgart 1976; Gerhard Lehmbruch, Parteienwettbewerb im Bundesstaat: Regelsysteme und Spannungslagen im politischen System der Bundesrepublik Deutschland, Wiesbaden 2000. 
gebunden. Der Parteienwettbewerb und ein hoher vertikaler Integrationsgrad der Partei$\mathrm{en}^{22}$ sorgen so für eine enge Kopplung der Arenen.

Deren Inkongruenz, von Gerhard Lehmbruch zunächst als „Strukturbruch“, später als „Verwerfung“ bezeichnet, birgt die Gefahr der Verzerrung von intergouvernementalen Verhandlungen. Wenn nun durch Gremienreduktion die politische Koordinierungsfunktion der Fachminister beziehungsweise ihrer Staatssekretäre und Abteilungsleiter an Bedeutung gegenüber den Koordinierungsleistungen der Verwaltungsebene gewinnt, weil AgendaSetting und Entscheidungsvorbereitung auf höherer hierarchischer Ebene erfolgen oder eine engere politische Steuerung beispielsweise durch konkrete Mandatierung oder Einrichtung von Ad-hoc-Arbeitsgruppen angestrebt wird (Politisierungsthese), dann führt dies auch zu einer engeren Kopplung der Koordinationsprozesse und -arenen an den Parteienwettbewerb. Dieser gewinnt für die bundesstaatliche Koordination an Bedeutung und erhöht die Gefahr für Spannungen und Konflikte. Die zweite Hypothese postuliert daher: Politisierung schwächt die bundesstaatliche Politikkoordination. Dies kann sich in einem höheren Zeitaufwand für den Koordinierungsprozess ausdrücken, zum Beispiel weil Vorschläge von Arbeitsgremien fachlich nochmals auf der politischen Ebene beraten werden. Die Koordination ist aber auch dann schwächer, wenn insgesamt weniger Politikergebnisse abgestimmt werden, wenn die erreichten Ergebnisse von schlechterer Qualität oder weniger umfassend sind, was ihre Inhalte oder die beteiligten Länder betrifft.

Den nachgeordneten Beamten der Ministerialbürokratie ist grundsätzlich daran gelegen, anfallende Verhandlungsgegenstände einvernehmlich auf der Arbeitsebene zu regeln. ${ }^{23}$ Sie arbeiten in sektoral orientierten „Fachbruderschaften "24 zusammen und versuchen unter verwaltungsfachlichen und Vollzugsgesichtspunkten geeignete Vorschläge und Programme zu erarbeiten, die häufig von der politischen Ebene ohne weitere Diskussion übernommen werden. ${ }^{25}$ Für die Arbeitsebene ist somit der kooperativ angelegte Verhandlungstypus des „Problemlösens“ der Regelfall, weil hier gemeinsame Interessen und Ziele im Mittelpunkt stehen. ${ }^{26}$ Für Verhandlungen im Schatten des Parteienwettbewerbs ist dagegen als vorherrschende Interaktionsorientierung eher ein „Bargaining-Modus“ zu erwarten, bei dem die Durchsetzung konkurrierender Interessen im Vordergrund steht. Die Politisierung der intergouvernementalen Koordination fördert Konkurrenzverhalten und Konfrontationsstrategien der beteiligten Akteure und führt tendenziell zu einer Umformung konkurrierender

22 Vgl. Edgar Grande, Parteiensystem und Föderalismus. Institutionelle Strukturmuster und politische Dynamiken im internationalen Vergleich, in: Arthur Benz / Gerhard Lehmbruch (Hrsg.), a.a.O. (Fn. 2), S. $179-212$.

23 Vgl. Wolfgang Renzsch, Konfliktlösung im parlamentarischen Bundesstaat, in: Rüdiger Voigt (Hrsg.), Der kooperative Staat. Krisenbewältigung durch Verhandlung?, Baden-Baden 1995, S. $167-192$, S. 185.

24 Vgl. Frido Wagener, Der öffentliche Dienst im Staat der Gegenwart, in: Vereinigung der Deutschen Staatsrechtslehrer (Hrsg.), Veröffentlichungen der Vereinigung der Deutschen Staatsrechtslehrer, Heft 37, Berlin / New York 1979, S. 215 - 266.

25 Vgl. Wolfgang Graf Vitzthum / Wolfgang März, Baden-Württembergs Stellung in der ZwischenLänder-Zusammenarbeit, in: Hartmut Klatt (Hrsg.), a.a.O. (Fn. 10), S. 147 - 174.

26 Vgl. zu den Verhandlungstypen Renate Mayntz, Policy-Netzwerke und die Logik von Verhandlungssystemen, in: Adrienne Héritier (Hrsg.), Policy-Analyse: Kritik und Neuorientierung, PVSSonderheft 24, Opladen 1993, S. 39 - 56; Fritz W. Scharpf, Interaktionsformen. Akteurzentrierter Institutionalismus in der Politikforschung, Wiesbaden 2006. 
Interessen, die mit Kompromissen ausgleichbar sind, in antagonistische Interessen, bei denen es zu Nullsummen-Verteilungskonflikten kommt. ${ }^{27}$

Die Problematik wird noch verschärft durch das der politischen Führung stets überlegene Fachwissen der Verwaltung ${ }^{28}$, das im Rahmen zentralisierter politischer Steuerung weder in umfassender Weise verfügbar gemacht noch vollständig sachgerecht verarbeitet werden kann, was die Entscheidungsqualität beeinträchtigt. ${ }^{29}$ Auch die im Beschluss der MPK angestrebte Reduktion von ständigen Einrichtungen - durch Auflösung oder Umwandlung in Ad-hoc-Gremien - kann sich nachteilig auf die Koordination auswirken. Auf Dauer angelegte Verhandlungssysteme erfordern einen ebensolchen institutionellen Rahmen, da in ihm „nicht nur über je besondere, sondern auch über gemeinsame Interessen informiert, verhandelt und fair entschieden werden kann"30. Entfällt dieser dauerhafte institutionelle Rahmen, so fehlt den Akteuren ein wichtiger Ort für Vertrauensbildung und offenen Informationsaustausch - beides grundlegende Voraussetzungen für das kooperative „Problemlösen“.

Die Politisierung von Koordination im deutschen Bundesstaat verschiebt somit die Grenzen zwischen Parteipolitik und fachlichen Vollzugsfragen, sie schwächt den problemlösungsorientierten Verhandlungsmodus und verstärkt die kompetitiven Handlungsorientierungen der beteiligten Akteure. Diese Entwicklung erschwert die Erarbeitung gemeinsamer Problemlösungen und schwächt eine effektive Koordination und Problembearbeitung im Bundesstaat.

\subsection{Persistenz und Informalisierung}

Die bisherigen Überlegungen gehen davon aus, dass die Fachministerkonferenzen den Beschluss der MPK umgesetzt, das heißt Gremien in signifikantem Ausmaß abgeschafft und Koordinationsprozesse möglichst auf befristete Projektgremien verlagert haben. Eine buchstabengetreue Durchführung des Beschlusses und ein umfangreicher Abbau von Gremien der bundesstaatlichen Koordinierung erscheinen jedoch aus mehreren Gründen recht unwahrscheinlich. Zunächst einmal bleibt die oben bereits angesprochene Komplexität der Informationen und Regelungsinhalte bestehen, zu deren Bearbeitung die politische Führung insbesondere in sehr fachlich-technisch geprägten Bereichen auf die vorbereitenden und strukturierenden Funktionen der Verwaltung angewiesen ist. Dazu konstatierte Fritz Scharpf bereits zu Beginn der 1970er Jahre, dass „die formelle Verlagerung der Entschei-

27 Vgl. Arthur Benz, Mehrebenen-Verflechtung. Verhandlungsprozesse in verbundenen Entscheidungsarenen, in: ders. / Fritz W. Scharpf/ Reinhard Zintl (Hrs.), Horizontale Politikverflechtung. Zur Theorie von Verhandlungssystemen, Frankfurt am Main / New York 1992, S. 147 - 205, S. 161.

28 Vgl. Renate Mayntz, Soziologie der öffentlichen Verwaltung, Heidelberg / Karlsruhe 1978; Max Weber, Wirtschaft und Gesellschaft, Tübingen 1976.

29 Vgl. Fritz W. Scharpf, Planung als politischer Prozeß. Aufsätze zur Theorie der planenden Demokratie, Frankfurt am Main 1973, S. 84 f.; Fritz W. Scharpf/ Bernd Reissert / Fritz Schnabel, Politikverflechtung. Theorie und Empirie des kooperativen Föderalismus in der Bundesrepublik, Kronberg im Taunus 1976, S. 28 f.

30 Fritz W. Scharpf, Koordination durch Verhandlungssysteme: Analytische Konzepte und institutionelle Lösungen, in: Arthur Benz / Fritz W. Scharpf / Reinhard Zintl (Hrsg.), a.a.O. (Fn. 27), S. $51-96$, S. 91 . 
dungskompetenz nach oben an der materiellen Dezentralisierung des Problemverarbeitungsprozesses wenig oder nichts ändert" ${ }^{31}$. Da von Politikern, Bürgern und Unternehmen gleichermaßen ein möglichst einheitlicher Gesetzesvollzug im gesamten Bundesgebiet erwartet und gefordert wird, bleibt die Verwaltung auf eine Vorab-Koordination der sechzehn Länder mit dem Bund angewiesen. Koordinierende Arbeitsgremien sind daher unter den Voraussetzungen des unitarischen Föderalismus („gleichwertige Lebensverhältnisse“) aus einer funktionalen Perspektive unabdingbar.

Einem umfassenden Gremienabbau stehen auch die Beharrungskräfte und das institutionelle Eigeninteresse der politisch-administrativen Institutionen entgegen. Bürokratische wie politische Akteure neigen dazu, ihre individuellen und persönlichen Interessen mit dem Erhalt und möglichst der Ausweitung von Funktionen und der Stellung jener Organisationen zu verknüpfen, denen sie angehören. Für die Handelnden verbindet sich ihr Interesse an Karrierechancen, Einkommen, sozialen Netzwerken sowie persönlicher Entfaltung mit dem organisationsbezogenen Interesse an umfangreichen sektoralen Programmen und Ressourcen. ${ }^{32}$ Die „Verteidigung der institutionellen Eigeninteressen (wird) deshalb zu einem besonders schwer überwindbaren Hindernis der staatlichen Politik“33. Eine Beschränkung der administrativen Koordination ist unter diesen Voraussetzungen am wahrscheinlichsten für Gremien, die auf eine eher kurze „Tradition“ zurückblicken, die von den zu bearbeitenden Inhalten eher politisch-programmatisch als fachlich-technisch orientiert sind oder deren Themen gerade nicht prominent auf der aktuellen politischen Tagesordnung stehen.

Damit ist jedoch noch nicht die Frage geklärt, ob Gremienreduktion nicht allein formal, sondern auch de facto gelingen kann. Aufgrund der funktionalen Erfordernisse und der Beharrungskräfte sektoraler Fachbruderschaften können die Akteure in solchen Bereichen, in denen Koordinationsprozesse durch Gremienreduktion erschwert worden sind, möglicherweise auf alternative Strategien ausweichen. Dazu gehören die Koordination in der Fachgemeinschaft außerhalb offizieller Arbeitsgremien oder -aufträge und die eher infor-

\begin{tabular}{|lll|}
\hline Abbildung 1: Gremienreduktion und ihre Auswirkungen im deutschen Bundesstaat \\
\cline { 2 - 3 } Gremienreduktion & Politisierung & $\begin{array}{l}\text { Bundesstaatliche } \\
\text { Politikkoordination }\end{array}$ \\
- Auflösung oder & - formal & - Schwächung \\
- Befristung & - funktional & \\
- Tagungshäufigkeit & & \\
- Mandatierung \\
personelle Besetzung
\end{tabular}

31 Fritz W. Scharpf, a.a.O. (Fn. 29), S. 85.

32 Diese Argumentation findet sich konkret bei Anthony Downs, Inside Bureaucracy, Boston 1967, S. 81 - 87; oder ähnlich Fritz W. Scharpf, Grenzen der institutionellen Reform, in: Thomas Ellwein / Joachim Jens Hesse / Renate Mayntz / ders. (Hrsg.), Jahrbuch zur Staats- und Verwaltungswissenschaft, Bd. 1, Baden-Baden 1987, S. $111-151$.

33 Ebenda, S. 123. 
melle Abstimmung auf Arbeitsebene. Es ist daher durchaus vorstellbar, dass die langjährigen Verwaltungsnetzwerke weiter bestehen bleiben und sich sogar - anders als die Politisierungsthese vermutet - weiter verselbständigen und der Kontrolle der politischen Ebene entziehen. Entsprechend lautet die dritte Hypothese: Gremienreduktion stärkt informale Verwaltungsnetzwerke und kann zu ihrer Entkopplung von der Politik führen (Informalisierungsthese).

\section{Empirische Umsetzung}

Die MPK übermittelte ihren Beschluss zur Gremienreduktion mit Schreiben vom 5. Juli 2004 an die Fachministerkonferenzen, da der Beschluss ressortweise umzusetzen war. Die vergleichende Betrachtung des Gremienabbaus über alle Fachministerkonferenzen wird allerdings durch zwei Faktoren erschwert: Erstens sind Informationen zu den jeweils vorhandenen Gremien nur für einen Teil der Konferenzen öffentlich zugänglich; andere Ressorts behandeln Entscheidungen über Arbeitsgremien und -strukturen dagegen als vertraulich und nicht-öffentlich. Zweitens schränkt die schiere Anzahl der Gremien auf verschiedenen Ebenen und mit variierendem Zuschnitt die Möglichkeiten ein, umfassende Übersichten für einen bestimmten Zeitraum zu erstellen.

Tabelle 1 ist in erster Linie auf Basis der von der MPK gesammelten Unterlagen, das heißt den Meldungen der einzelnen Fachministerkonferenzen zum Gremienabbau, entstanden und ermöglicht einen eingeschränkten Vergleich zwischen Politikfeldern. Der Vergleich ist deshalb nur eingeschränkt möglich, weil die Meldungen an die MPK nicht auf einem einheitlichen Maßstab beruhten, so zum Beispiel häufig nur die Gremien der obersten Arbeitsebene (Abteilungsleiterebene) gemeldet wurden oder formal befristete Gremien keine Berücksichtigung fanden. ${ }^{34}$ Die erwähnten Probleme des Zugangs zu ergänzenden Daten ermöglichten keine weitergehende Vereinheitlichung der Angaben.

Die rechte Spalte der Tabelle zeigt, dass bereits 1998/99 ein Gremienabbau in erheblichem Umfang stattgefunden hat. Insbesondere aus den Bereichen Verkehr (VMK), Landwirtschaft (AMK), Umwelt (UMK) und Finanzen (FMK) wurde eine deutliche Reduktion gemeldet. Diese Anstrengungen zum Gremienabbau etwa fünf Jahre vor einer erneuten Initiative der MPK stellen einen wichtigen Kontextfaktor für die späteren Reduktionsentscheidungen der Fachministerkonferenzen dar. Ebenso ist die Gremienstruktur der einzelnen Konferenzen im Jahr 2004 ein relevanter Faktor für die Bewertung des Gremienabbaus im darauf folgenden Zeitraum. Aufgrund der uneinheitlichen Datenlage nennt die Tabelle hier keine absoluten Zahlen; es war jedoch möglich, die Fachministerkonferenzen in Beziehung zueinander zu setzen und so relative Angaben zu machen. Diese Beschreibung des „Ausgangszustandes“ deutet darauf hin, dass das Potential für eine erneute Gremienreduktion zwischen den Ressorts allein aufgrund der noch vorhandenen Zahl der Gremien variierte, wobei ebenfalls zu berücksichtigen ist, dass sich auch der tatsächliche Koordinationsbedarf beträchtlich unterscheiden dürfte und damit auch unterschiedliche institutionelle Strukturen erfordert. Darüber hinaus fällt auf, dass ein Teil der Ressorts trotz deutlicher

34 Nachgeordnete Gremien wurden häufig als nicht relevant im Sinne des MPK-Beschlusses angesehen. Möglicherweise lagen einigen Konferenzen auch selbst keine umfassenden Übersichten über die Gremien in ihrem Arbeitsbereich vor. 


\begin{tabular}{|c|c|c|c|c|c|c|c|c|c|c|c|c|c|c|c|}
\hline & 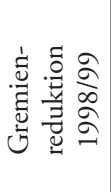 & $\infty$ & 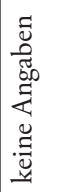 & $\beth$ & 0 & $\hat{0}$ & 0 & $\stackrel{\sim}{\sim}$ & $\stackrel{\infty}{\sim}$ & 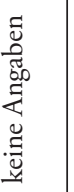 & 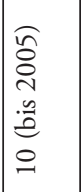 & $\sigma$ & 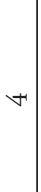 & 0 & న \\
\hline & 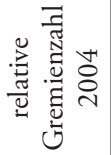 & 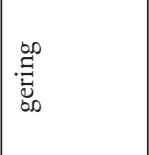 & 莺 & $\begin{array}{l}\tilde{\mathcal{Z}} \\
0\end{array}$ & 点 & $\stackrel{\overrightarrow{\underline{\Xi}}}{\vec{g}}$ & 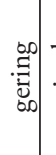 & 䳮 & $\begin{array}{l}\tilde{\Xi} \\
0 \\
\end{array}$ & 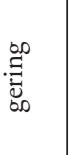 & 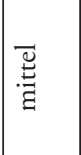 & 离 & 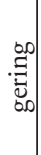 & : & 离 \\
\hline & 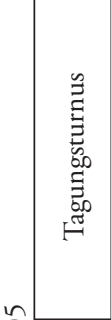 & 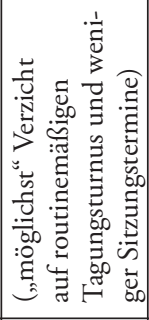 & 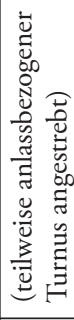 & 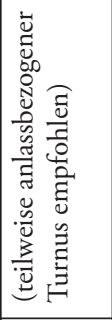 & 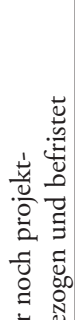 & & & 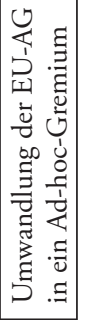 & & 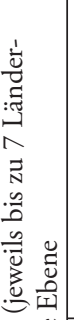 & 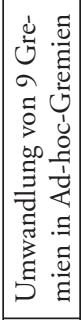 & 1 & 1 & 1 & 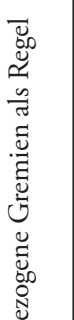 \\
\hline $\begin{array}{l} \\
\text { ป } \\
\text { ปे } \\
\text { है } \\
\text { है }\end{array}$ & 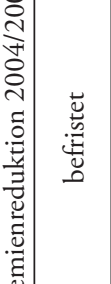 & 1 & 1 & 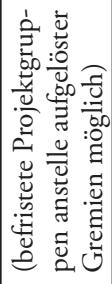 & 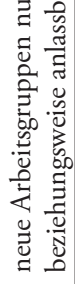 & & & 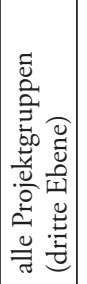 & & 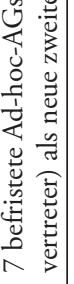 & I & 1 & 1 & 1 & 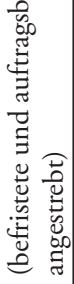 \\
\hline 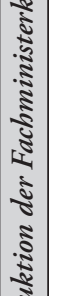 & 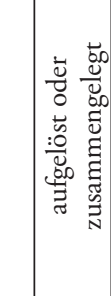 & 1 & 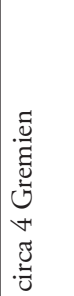 & 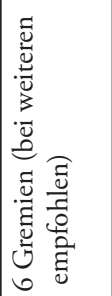 & 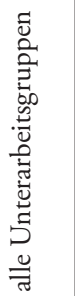 & 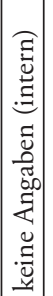 & & 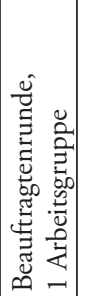 & 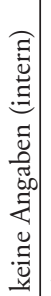 & 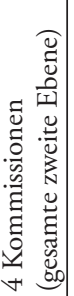 & 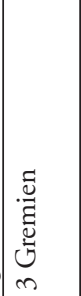 & 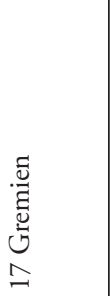 & & $*_{1}$ & 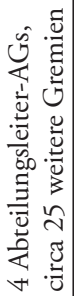 \\
\hline 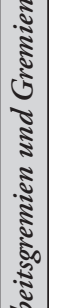 & 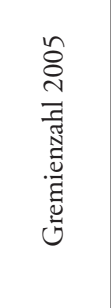 & 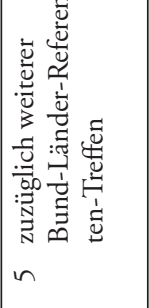 & 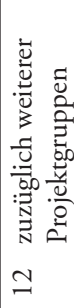 & $\stackrel{\circ}{\sim}$ & 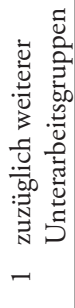 & 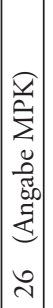 & $\operatorname{nn}$. & $\stackrel{m}{2}$ & 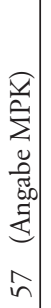 & $\infty$ & $\stackrel{\sim}{\sim}$ & 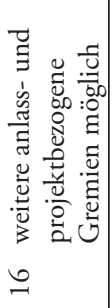 & in & ○ & $\stackrel{\sim}{F}$ \\
\hline 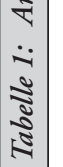 & 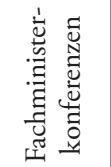 & $\sum_{<}^{2}$ & 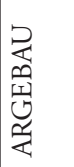 & 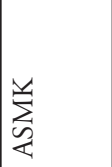 & $\sum_{i=1}^{n}$ & $\sum_{i=1}^{4}$ & 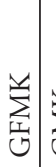 & $\sum_{\cup}^{*}$ & $\sum$ & $\sum$ & $\sum_{\underline{2}}^{0}$ & $\sum$ & $\frac{\pi}{\Sigma}$ & 心) & $\sum_{S}^{J}$ \\
\hline
\end{tabular}




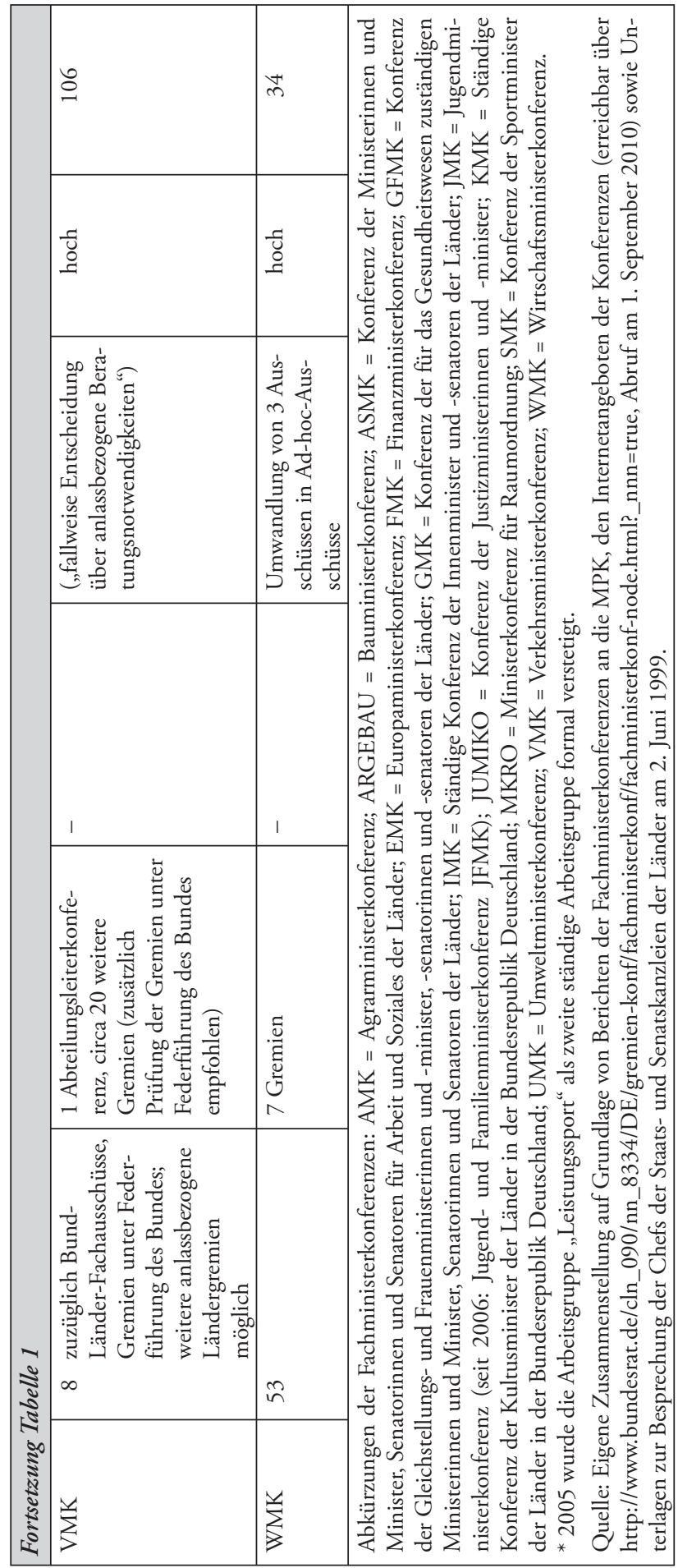


Reduktion 1998/99 auch weiterhin, zumindest bis zum Jahr 2004, über eine relativ hohe Gremienzahl verfügte. Eine hohe Gremienzahl kann einerseits wohl stets mit einem hohen faktischen Koordinationsbedarf in differenzierten Themenfeldern erklärt werden; andererseits ist es auch möglich, dass in der Zeit nach dem Abbau Ende der 1990er Jahre wieder eine Reihe neuer Gremien eingerichtet wurden. Diese Annahme stützt sich auf die Aussage eines Staatskanzlei-Mitarbeiters, der davon ausgeht, ein solcher Abbau müsse alle fünf Jahre wiederholt werden, da die Gremien sonst überhand nähmen. ${ }^{35}$

Die Variable "Gremienreduktion" ist definiert durch ihre empirisch beobachteten Dimensionen (vgl. Abbildung 1), das heißt der nach dem MPK-Beschluss von 2004 umgesetzten Maßnahmen. Dazu gehörte, dass Gremien aufgelöst beziehungsweise zusammengelegt sowie zeitlich befristet wurden und/oder nur noch projekt- oder anlassbezogen (ad hoc) oder zumindest weniger häufig tagen sollten. ${ }^{36}$ Auch hier bilden die mittleren Spalten der Tabelle wieder eine deutliche Varianz ab. Während in einigen Politikfeldern eine relativ umfangreiche Gremienreduktion erfolgt ist, hat es in anderen Bereichen gar keine Veränderungen gegeben. Die Ministerkonferenzen in den Bereichen Landwirtschaft (AMK), Gleichstellung und Frauen (GFMK), Raumordnung (MKRO) sowie Sport (SMK) teilten der MPK mit, dass die vorhandenen Gremien bereits als das Mindestmaß für die erforderliche bundesstaatliche Koordinierung betrachtet würden. Eine (weitere) Reduzierung wurde weder als notwendig noch als im Rahmen der Aufgabenerfüllung umsetzbar angesehen. Ähnlich argumentierte auch die Innenministerkonferenz (IMK); die SMK verstetigte sogar 2005 in ihrer Geschäftsordnung die Arbeitsgruppe Leistungssport zu einem ständigen Gremium.

Einen deutlichen Abbau haben dagegen durchaus auch solche Fachministerkonferenzen durchgeführt, die bereits einige Jahre zuvor signifikant reduziert hatten oder wo bereits vorher eine relativ geringe Gremienausstattung vorhanden war. So hat die Jugendministerkonferenz (JMK) ihre gesamte zweite Arbeitsebene aufgelöst und durch befristete Ad-hocArbeitsgemeinschaften ersetzt. Diese sind jeweils mit bis zu sieben Landesvertretern besetzt und können damit gar keinen Konsens mehr zwischen allen Ländern auf dieser Ebene herstellen. Ähnlich sind eine Reihe weiterer Konferenzen auf einer untergeordneten Arbeitsebene vorgegangen, indem bisherige Dauergremien durch befristete Projektgruppen ersetzt und nur noch mit konkreten, von der politischen Ebene genehmigten oder erteilten Aufträgen befasst werden. Zu dieser Gruppe gehören die Europaministerkonferenz (EMK) wie auch die Gesundheitsministerkonferenz (GMK), die zudem ihre „Beauftragtenrunde“ abgeschafft hat, die ein auch personelles Bindeglied zwischen der fachlichen und politischen Vorbereitung der GMK-Sitzungen bildete. ${ }^{37}$ Gremienreduktion führt so zu einer strikteren

35 Die empirischen Fallbeispiele stützen sich auf Dokumentenanalysen sowie mehrere Experteninterviews, die zwischen dem 15. Juli 2008 und dem 29. Juli 2009 in Berlin, Bonn und Düsseldorf durchgeführt wurden. Meinen Gesprächspartnern bin ich für die freundliche Auskunftsbereitschaft zu Dank verpflichtet.

36 Informationen zu einer verstärkten Mandatierung und zu Vorgaben der personellen Besetzung liegen nur in Einzelfällen vor und wurden daher nicht in die Tabelle aufgenommen.

37 Die Beauftragtenrunden dienten seit 1998 der Vorbereitung politisch bedeutsamer Themen für die Beratung in der GMK. Die fachliche Zusammenarbeit der Länder sollte daneben auf Abteilungsleiterebene in der Arbeitsgemeinschaft der Obersten Landesgesundheitsbehörden (AOLG) erfolgen, wobei zwischen beiden Gremien Personalidentität zulässig war. Seit 2005 werden die Sitzungen der GMK nun ausschließlich durch eine Amtschefkonferenz der Staatssekretäre vorbereitet, während die AOLG weiterhin die fachlichen Themen bearbeitet. 
Trennung zwischen fachlichen und politischen Koordinationsaufgaben, was fachliche Initiativen der Arbeitsebene erschwert und sehr wahrscheinlich die bundesstaatliche Koordination schwächt.

\section{Fallbeispiele aus dem Umweltschutz}

Die Umweltpolitik eignet sich aus zwei Gründen für eine detailliertere empirische Analyse. Erstens hat die Umweltministerkonferenz (UMK) nach dem MPK-Beschluss auf allen Ebenen Gremien aufgelöst und zusammengelegt; auch 1998/99 wurde hier bereits deutlich reduziert. Zweitens handelt es sich bei der Umweltpolitik tendenziell um ein Politikfeld mit starkem Parteienwettbewerb ${ }^{38}$, so dass hier die Auswirkungen der Politisierungsthese und eine Schwächung der Koordination nachvollziehbar sein sollten.

Diskussionen um Struktur und Erforderlichkeit der im Umweltbereich bestehenden Gremien waren bereits vor dem MPK-Beschluss von 2004 geführt worden. Als dann die Ministerpräsidenten eine deutliche Reduktion forderten, stand die UMK diesem Ziel grundsätzlich positiv und aufgeschlossen gegenüber; über die konkrete Umsetzung entschieden die vorbereitenden Staatssekretärsrunden (Amtschefkonferenz, ACK). Die Neustrukturierung der ersten Arbeitsebene sowie grundsätzliche Vorgaben zur Struktur der nachfolgenden Ebenen wurden von der ACK am 21. Oktober 2004 beschlossen, in leicht veränderter und erweiterter Form dann von der UMK am 4./5. November 2004 bestätigt. Über die ständigen Ausschüsse auf der zweiten Arbeitsebene entschied die ACK nach Konsultation der Bund-Länder-Arbeitsgemeinschaften am 18./19. Mai 2005, die UMK bestätigte das Votum am 19./20. Mai 2005.39

Damit wurde im Umweltbereich die Zahl der Arbeitsgruppen insgesamt stark reduziert. Auf der ersten Arbeitsebene sank ihre Zahl von zwölf auf acht. Die Aufgaben der aufgelösten Gremien wurden teilweise auf andere übertragen, ein Arbeitskreis wurde auf die darunterliegende Ebene verschoben. 2004 bestanden darüber hinaus noch 61 Ausschüsse sowie Unterausschüsse und Arbeitsgruppen auf den darunterliegenden Ebenen. Nach Umsetzung des MPK-Beschlusses verblieben stattdessen 33 untergeordnete Gremien. Die gesamte UMK-Gremienstruktur besteht nun aus maximal vier Ebenen: Die politische Leitungsfunktion liegt weiterhin bei UMK und ACK. Darunter sind die acht Bund-Länder-Arbeitsgemeinschaften angesiedelt, deren Mitglieder nun grundsätzlich Abteilungsleiter sein müssen. Zuvor waren in den Gremien auf dieser Ebene häufig auch Referatsleiter vertreten. ${ }^{40}$ Den Bund-Länder-Arbeitsgemeinschaften sind ständige Ausschüsse zugeordnet, wobei deren Zahl so gering wie möglich gehalten werden soll. In der Regel sollen stattdessen im Bedarfsfall sogenannte Ad-hoc-Ausschüsse eingerichtet werden, deren Laufzeit auf maximal ein Jahr zu befristen ist und deren Einrichtung beziehungsweise Weiterführung von der

38 Vgl. Gisela Müller-Brandeck-Bocquet, Die institutionelle Dimension der Umweltpolitik, BadenBaden 1996; Axel Volkery, Naturschutzpolitik in den Bundesländern, in: Achim Hildebrandt I Frieder Wolf(Hrsg.), Die Politik der Bundesländer: Staatstätigkeit im Vergleich, Wiesbaden 2008, S. $257-273$.

39 Die Ergebnisprotokolle aller UMK- und ACK-Sitzungen seit 1998 sind online zugänglich unter http://www.umweltministerkonferenz.de (Abruf am 14. Juli 2010).

$40 \mathrm{Vgl}$. Sachverständigenrat für Umweltfragen, Umweltverwaltungen unter Reformdruck. Herausforderungen, Strategien, Perspektiven - Sondergutachten, Berlin 2007, S. 104. 
ACK genehmigt werden muss. Die Einrichtung neuer Arbeitsgremien erfordert in jedem Fall die Zustimmung von ACK oder UMK; zudem dürfen die Gremien nur noch im Rahmen von (grundsätzlichen oder konkreten) Aufträgen der politischen Leitungsebene tätig werden.

\subsection{Beispiel Nachhaltige Entwicklung}

Im Rahmen der Umsetzung der Nationalen Nachhaltigkeitsstrategie der Bundesregierung richtete die UMK 2001 einen Bund-Länder-Arbeitskreis Nachhaltige Entwicklung ein. 2005 wurden alle von der UMK aufgelösten Arbeitsgremien der ersten Ebene in die dann sogenannte Bund-Länder-Arbeitsgemeinschaft Nachhaltige Entwicklung (BLAG NE) integriert. Die AG war damit zusätzlich zu ihrem ursprünglichen Auftrag zuständig für die Themenbereiche fachübergreifende Umweltangelegenheiten, Energie, Klimaschutz und Klimafolgen, Verkehr, steuerliche und wirtschaftliche Fragen des Umweltschutzes sowie Umweltinformationssysteme. Letzteres wurde in einen ständigen Ausschuss ausgegliedert; zudem richtete man Ad-hoc-Ausschüsse ein, unter anderem für „Klimaschutz, Energie und Verkehr“. 2007 wurde die Thematik des Klimaschutzes und -wandels aufgewertet, indem der Ad-hoc-Ausschuss formal in eine neue Bund-Länder-Arbeitsgemeinschaft (BLAG $\mathrm{KliNa}=$ Klima, Energie, Mobilität - Nachhaltigkeit) umgewandelt wurde, in der wiederum die BLAG NE aufging (69. UMK, TOP 28). Als bisher letzte Änderung wurde das Klimathema im Juni 2009 erneut ausgelagert, als die UMK die Einrichtung eines neuen ständigen Ausschusses „Anpassung an die Folgen des Klimawandels - AFK“ genehmigte (72. UMK, TOP 9).

Aus der Zusammenlegung der verschiedenen Themenbereiche und den Vorgaben der UMK folgte notwendigerweise eine personelle Umbesetzung der AG Nachhaltige Entwicklung. Anstelle von vier separaten Arbeitskreisen mit den jeweils fachlich zuständigen Vertretern der Länder (in der Regel Referatsleiter) gab es nur noch den mit jeweils einem Abteilungsleiter aus jedem Land besetzten BLAG NE. Daraus ergaben sich zunächst vor allem Schwierigkeiten der Arbeitsorganisation. Man musste erst einmal recht mühsam zu einem gemeinsamen Gremium und Aufgabenverständnis zusammenfinden. Die jeweiligen Vertreter im BLAG NE hatten nun außerdem in den Sitzungen thematisch einen sehr breiten Bereich abzudecken, was auch in umfangreicheren Tagesordnungen resultierte. Dennoch wurde zusätzlich noch das als aktuell und politisch dringlich eingestufte Klimathema aus dem befristeten Ad-hoc-Ausschuss heraus verstetigt und auf die BLAG-Ebene verlagert (69. UMK, TOP 28).

Für die Thematik der Nachhaltigkeit bedeutete die Zusammenlegung mit anderen Arbeitskreisen zu einem sehr breit angelegten Gremium eine organisatorische Schwächung, obwohl letztlich nach kontroversen Diskussionen die Untergremien („Indikatoren“ sowie „Bildung für nachhaltige Entwicklung") als Ad-hoc-Ausschüsse in ihrer bestehenden personellen Struktur beibehalten wurden. Für die Effektivität der Koordination war sie dennoch ein - nach Einschätzung eines Beteiligten - „extremer“ Nachteil: Innerhalb des neuen BLAG NE beziehungsweise BLAG KliNa kam es zu einer thematischen Akzentverschiebung; das Thema Nachhaltigkeit verlor hier und in der Folge auch in der UMK und den Umweltministerien der Länder an Gewicht. Die Verlagerung der Diskussionen in der AG von Referatsleitern auf die Abteilungsleiter der Länder bedeutete einen Bruch in der Konti- 
nuität, die resultierende Koordination ist weniger umfassend. Zu dieser Entwicklung haben vermutlich der Querschnittscharakter der Thematik, die politisch-programmatische Ausrichtung des Gremiums und die noch wenig ausgeprägte Tradition als Bund-Länder-Gremium beigetragen. Das Thema Klimawandel nahm dagegen eine andere Entwicklung: Es wurde auf Initiative des BLAG KliNa inzwischen in einen neuen ständigen Ausschuss verlagert, weil sich erwiesen hatte, dass es kontinuierlich und nicht nur befristet beraten werden muss, die alleinige Beratung auf Abteilungsleiterebene aufgrund der fachlichen Komplexität aber ebenfalls nicht ausreichte.

\subsection{Beispiel Umweltinformatik}

Der frühere Bund-Länder-Arbeitskreis Umweltinformationssysteme (BLAK UIS) wurde im Zuge des Beschlusses zur Gremienreduktion als ständiger Ausschuss (StA UIS) des BLAG NE und später des BLAG KliNa beibehalten. Sämtliche Ausschüsse des ursprünglichen BLAK wurden jedoch gestrichen, ihre Aufgaben teilweise vom StA UIS übernommen. Die personelle Besetzung des Ausschusses hat sich nicht verändert, die Länder sind weiterhin auf Referatsleiterebene vertreten.

Im Bereich Umweltinformatik sind geringere Auswirkungen der Gremienreduktion zu beobachten. Die Koordination hat sich jedoch „verkompliziert“; sie ist zeitaufwändiger geworden, weil Arbeitsergebnisse von den übergeordneten Ebenen genehmigt werden müssen und sich dabei wegen der indirekteren Kommunikationswege Verständnisschwierigkeiten oder inhaltliche Verfälschungen ergeben können. Darüber hinaus haben sich aber die Koordinationsprozesse in diesem Bereich kaum verändert. Eine Erklärung dafür ist der ausgesprochen technische Charakter der Themen des Ausschusses, die offenbar derzeit für den politischen Wettbewerb nicht relevant sind. So werden zum Beispiel Berichte des Ausschusses in der Regel problemlos von der politischen Ebene übernommen. Für diese Erklärung spricht auch, dass die Arbeit des Ausschusses durch seine neue hierarchische Einordnung eher entpolitisiert und eben gerade nicht von den Abteilungsleitern übernommen werden sollte.

Am Beispiel des StA UIS lassen sich Ausweichstrategien der administrativen Akteure nachvollziehen: Als ein vom StA UIS angestrebter Ad-hoc-Ausschuss zum Thema Datenaustausch nicht genehmigt wurde, führte man stattdessen einen „Erfahrungsaustausch“ unter Beteiligung einiger Länder durch. Es fanden zwei kurze Treffen statt, die weitere Abstimmung erfolgte jedoch hauptsächlich per E-Mail. Die beteiligten Akteure fanden also eine Möglichkeit, die institutionalisiertere Form der Koordination in einem Ad-hoc-Ausschuss durch eine informellere Alternative zu ersetzen, jedoch war es „extrem schwierig, (letztlich ein) Ergebnis herbeizuführen, weil wir nicht die Möglichkeit hatten, (...) uns wirklich dann auch mal zwei, drei Tage hinzusetzen und das Thema durchzudiskutieren“. Im Zuge der Umsetzung des MPK-Beschlusses wurde die erlaubte Tagungsdauer für die in halbjährlichem Turnus stattfindenden Sitzungen des UIS-Ausschusses von drei auf zwei Tage reduziert. Da aber zusätzlich ein gesonderter Planungsausschuss aller Länder existiert, in dem über die Finanzierung konkreter gemeinsamer Projekte beraten wird und dessen personelle Besetzung derjenigen des StA UIS entspricht, tagt dieser nun seitdem gleich vor den StA-UIS-Sitzungen, was aus fachlicher Sicht eine insgesamt ausreichende Tagungsdauer ermöglicht. 
Für den Bereich Umweltinformationssysteme haben sich in der direkten fachlichen Zusammenarbeit vor allem deshalb keine Veränderungen ergeben, weil weiterhin dieselben Personen an der Koordinierung beteiligt sind. Hier lässt sich gut die Persistenz einer Fachbruderschaft beobachten, für die sich offenbar falls erforderlich auch flexible Ausweichstrategien finden, die jedoch in ihren Ergebnissen unterschiedlich einzuschätzen sind.

\subsection{Beispiel Hochwasserschutz}

Die Bund-Länder-Arbeitsgemeinschaft Wasser (LAWA) wurde von der UMK auf der ersten Arbeitsebene beibehalten. Sie verfügte bis 2005 über fünf ständige Ausschüsse, von denen drei im Zuge der Umstrukturierungen erhalten blieben ${ }^{41}$ und zwei wegfielen. Die Aufgaben dieser beiden („Anlagenbezogener Gewässerschutz“ sowie „Mengenmanagement und Informationsgrundlagen“) wurden auf die drei übrigen Ausschüsse aufgeteilt. Außerdem richtete die LAWA zwei Ad-hoc-Arbeitsgruppen zu den Themen „Hochwasser“ und „EUAbstimmung" ein. Bei der Umsetzung erschien in der LAWA insbesondere die Einordnung des Hochwasserschutzes problematisch, da die eigentlich zuständigen Fachleute aufgrund der Umstrukturierungen weder im Ausschuss für Grund- noch für Oberflächenwasser beteiligt sein würden, aber auch kein weiteres ständiges Gremium eingerichtet werden konnte. Daher wurde schließlich ein Ad-hoc-Ausschuss „Hochwasser“ mit einer Befristung auf zwei Jahre gegründet. Die so organisierte länderübergreifende Zusammenarbeit wurde noch 2006 von der UMK als effektiv und geeignet bezeichnet (66. UMK, TOP 4), dann jedoch im Juni 2008 ein ständiger Ausschuss „Hochwasserschutz und Hydrologie“ eingesetzt (70. UMK, TOP 17) und die Thematik damit unterhalb der Abteilungsleiterebene verstetigt.

Die Bearbeitung von Hochwasserschutzfragen in einem befristeten und auch rein formal auf die Bearbeitung konkreter Aufträge von UMK oder ACK beschränkten (GO UMK, Punkt 11.1) Ad-hoc-Gremium war problematisch für die bundesstaatliche Koordinierung, weil in diesem Rahmen nicht alle relevanten Fragen kontinuierlich und auf Dauer bearbeitet werden konnten. Die Mitbehandlung der Themen in einem anderen Ausschuss hätte jedoch wiederum das Problem der Vertretung mehrerer sehr technisch geprägter Bereiche durch einen Fachmann bedeutet, so dass man in der LAWA zunächst mit einem Ad-hocAusschuss arbeitete. Die Umstrukturierung wurde aber nicht beibehalten; die Fachbruderschaft hat erneut einen dauerhaften institutionellen Rahmen erhalten.

\section{Differenzierte Auswirkungen auf die bundesstaatliche Koordination}

Der Beschluss der MPK, der die Fachministerkonferenzen der Länder aufforderte, möglichst alle ihnen zuarbeitenden Gremien aufzulösen und Abstimmungen zukünftig in der Regel nur noch projektbezogen in befristeten Gremien durchzuführen, wurde in verschiedenen Politikfeldern ganz unterschiedlich umgesetzt. In einigen wurden keine Gremien abgebaut, andere Fachministerkonferenzen haben dagegen umfangreichere Strukturrefor-

41 Beibehalten wurden die Ausschüsse „Wasserrecht“, „Grundwasser und Wasserversorgung“ sowie „Oberirdische Gewässer und Küstengewässer“. 
men durchgeführt. Die Fallbeispiele aus dem Bereich des Umweltschutzes haben gezeigt, dass auch die Auswirkungen auf die bundesstaatlichen Koordinationsprozesse differenziert betrachtet werden müssen.

Die UMK hat in Reaktion auf den Beschluss der MPK ihre Arbeitsgremien relativ weitgehend reduziert, die neue Struktur stellt sich wesentlich „schlanker“ dar als zuvor. Eine verstärkte Tendenz zur Politisierung von Koordination ergibt sich aus der sehr grundlegenden Aufwertung der Abteilungsleiterebene wie auch der Beschränkung der nachgeordneten Gremien auf die Bearbeitung von Aufträgen der politischen Leitungsebene. Bei der Zusammenlegung von vorher eigenständigen Gremien zu einem neuen Gremium sind dort breitere Themen in der gleichen oder sogar kürzeren Zeit zu behandeln, in der Regel ergeben sich neue Gewichtungen zwischen den Themengebieten. Wenn Prioritäten gesetzt werden, wird die fachlich tiefe und umfassende Bearbeitung der übrigen Fragen erschwert. Weitere Folgen von Gremienreduktion sind ein erhöhter Zeitaufwand sowie Reibungsverluste bei einer hierarchisch gesteuerten inhaltlichen Abstimmung. Diese Beobachtungen weisen einerseits auf eine Schwächung der bundesstaatlichen Koordination hin. Andererseits haben die betroffenen Akteure auf der Arbeitsebene offenbar häufig die Möglichkeit, Ausweichstrategien anzuwenden, und es ist generell die Tendenz feststellbar, Strukturen mit einer gewissen personellen Kontinuität aufrecht zu erhalten. Wenn dies gelingt, zeigen sich nur wenige Veränderungen im eigentlichen Koordinationsprozess. In fachlich starken Bereichen mit einer langen Tradition der Koordinierung bleiben Gremien eher erhalten oder werden nur vorübergehend de-institutionalisiert; in diesen Fällen werden die Annahmen zur Persistenz sektoraler Fachbruderschaften bestätigt. Ob die hier am Beispiel der Umweltpolitik geschilderten Zusammenhänge auch für andere Politikfelder relevant sind, müssen weitere, vergleichend angelegte Untersuchungen zeigen. 